$i$. If $z=\sum_{i=1}^{n} t_{i} y_{i}$, then $z$ has the properties of the $z$ in the proof of Theorem 3.3, and it follows that $\|P\|=2$.

\title{
REFERENCES
}

1. R. S. Phillips, On linear transformations, Trans. Amer. Math. Soc. vol. 48 (1940) pp. 516-541.

2. A. Sobczyk, Projection of the space $(m)$ on its subspace $\left(c_{0}\right)$, Bull. Amer. Math. Soc. vol. 47 (1941) pp. 938-947.

UNIVERSITY OF TENNESSEE AND

Princeton University

\section{EXPANSIONS OF PARABOLIC WAVE FUNCTIONS}

\section{Y. ALAVI ${ }^{1}$ AND C. P. WELLS}

1. Introduction. The main result of this paper is an expansion of parabolic wave functions in a series of spherical wave functions with coefficients expressed in terms of Pasternack's functions. The series can be inverted, giving spherical wave functions as integrals of parabolic wave functions. Special cases include an expansion due to Hochstadt [1] and the limiting case where the wave functions become potential functions. We also give a new derivation of the bilinear generating function in the continuous case for the parabolic wave functions.

We call either $\psi(\xi, \lambda) \psi(\eta,-\lambda) e^{i \mu \phi}$, or simply $\psi(\xi, \lambda) \psi(\xi,-\lambda)$, where $\psi(\xi, \lambda)$ satisfies

$$
\xi^{2} \frac{d^{2} z}{d \xi^{2}}+\xi \frac{d z}{d \xi}+\left(k^{2} \xi^{4}+\lambda \xi^{2}-\mu^{2}\right) z=0,
$$

a parabolic wave function. The solution of (1) in which we are interested is given by

$$
\psi(\xi, \lambda)=\xi^{\mu} \exp \left(i k \xi^{2} / 2\right)_{1} F_{1}\left((w+\mu+1) / 2 ; \mu+1 ;-i k \xi^{2}\right),
$$

or in Whittaker's notation

$$
\psi(\xi, \lambda)=(-i k)^{-\mu / 2}\left(-i k \xi^{2}\right)^{-1 / 2} M_{-w / 2, \mu / 2}\left(-i k \xi^{2}\right),
$$

where $\lambda$ and $k$ are real and $w=-i \lambda / 2 k$. In physical applications $\mu$ is often an integer since then $\psi(\xi, \lambda)$ is regular and single valued

Presented to the Society, January 22, 1959; received by the editors November 17, 1958 and, in revised form, February 2, 1959.

1 The first named author is at Western Michigan University at the present time. 
for all $\xi$. However since the analysis is straightforward for arbitrary $\mu$ we shall not restrict it unless so indicated.

2. The expansion. We assume the expansion

$$
\psi(\xi, \lambda) \psi(\xi,-\lambda)=\sum_{n=0}^{\infty} a_{n+\mu}^{\mu} j_{n+\mu}(k r) P_{n+\mu}^{-\mu}(\cos \theta)
$$

where $j_{n+\mu}(k r)$ is a spherical Bessel function, $P_{n+\mu}^{-\mu}(\cos \theta)$ a Legendre function and the $a_{n+\mu}^{\mu}$ are to be determined. The reiations between spherical and parabolic coordinates give $2 r=\xi^{2}+\eta^{2}$ and $\cos \theta$ $=\left(\xi^{2}-\eta^{2}\right) /\left(\xi^{2}+\eta^{2}\right)$. Dividing both sides of (2) by $\xi^{\mu} \eta^{\mu}$ and letting $\xi, \eta \rightarrow 0$, we find $a_{\mu}^{\mu}=\Gamma(2 \mu+2) / k^{\mu}$.

Further if we divide (2) by $\eta^{\mu}$ and let $\eta \rightarrow 0$, we get

$$
\psi(\xi, \lambda) \Gamma(\mu+1)=\sum_{n=0}^{\infty} a_{n+\mu}^{\mu} \xi^{-\mu} j_{n+\mu}\left(k \xi^{2} / 2\right) .
$$

We now require that the right side of (3) be a solution of (1) and this leads to

$$
\sum_{n=0}^{\infty} a_{n+\mu}^{\mu}\left[v j_{n+\mu}^{\prime \prime}(v)+(1-\mu) j_{n+\mu}^{\prime}(v)+(v+i w) j_{n+\mu}(v)\right]=0
$$

with $v=k \xi^{2} / 2$. If we make use of the recursion formulas and differential equation for $j_{n+\mu}(v)$, we can reduce (4) to

$$
\begin{aligned}
\sum_{n=0}^{\infty} i^{n+1} \frac{\Gamma(n+2 \mu+1)}{\Gamma(n+1)} & j_{n+\mu}(v)\left[(n+\mu+1)(n+2 \mu+1) c_{n+\mu+1}^{\mu}\right. \\
& \left.+w(2 n+2 \mu+1) c_{n+\mu}^{\mu}-n(n+\mu) c_{n+\mu-1}^{\mu}\right]=0,
\end{aligned}
$$

where we have put $n ! a_{n+\mu}^{\mu}=(2 n+2 \mu+1) \Gamma(n+2 \mu+1) i^{n} c_{n+\mu}^{\mu}$. This implies that the $c_{n+\mu}^{\mu}$ satisfy

$$
\begin{aligned}
(n+2 \mu+1)(n+\mu+1) c_{n+\mu+1}^{\mu}+w(2 n+2 \mu & +1) c_{n+\mu}^{\mu} \\
& -n(n+\mu) c_{n+\mu-1}^{\mu}=0
\end{aligned}
$$

which is also the recursion formula for the functions $F_{n+\mu}^{\mu}(w)$ studied by Pasternack [2]. In terms of hypergeometric functions

$$
F_{n+\mu}^{\mu}(w)={ }_{3} F_{2}(-n-\mu, n+\mu+1,(1+w+\mu) / 2 ; 1,1+\mu ; 1)
$$

and is defined for all $\mu$ except negative integral values. Moreover

$$
\begin{aligned}
F_{\mu}^{\mu}(w) & ={ }_{2} F_{1}(-\mu,(1+w+\mu) / 2 ; 1 ; 1) \\
& =\frac{\Gamma[(1+\mu-w) / 2]}{\Gamma(\mu+1) \Gamma[(1-\mu-w) / 2]} .
\end{aligned}
$$


We choose that solution of (5) for which

$$
\frac{\stackrel{\mu}{c_{n+\mu}}}{c_{\mu}^{\mu}}=\frac{F_{n+\mu}^{\mu}}{F_{\mu}^{\mu}},
$$

and find the coefficients of (2) given by

(6) $\Gamma(n+1) \frac{{\stackrel{\mu}{a_{n+\mu}}}_{a_{\mu}^{\mu}}^{\mu}}{a_{\mu}}(2 n+2 \mu+1) \frac{\Gamma(n+2 \mu+1)}{\Gamma(2 \mu+2)} i^{n} \frac{F_{n+\mu}^{\mu}(w)}{F_{\mu}^{\mu}}$.

The convergence of (2) is assured for all values of the variables since for large $n, F_{n+\mu}^{\mu}(w)=O\left(n^{a}\right), P_{n+\mu}^{-\mu}(\cos \theta)=O\left(n^{b}\right)$, with $a$ and $b$ independent of $n$, while $j_{n+\mu}(k r)=O(1 / \Gamma(n+\mu+1))$.

Although an alternative derivation ${ }^{2}$ of (2) can be given the above method has the advantage of being applicable to other expansion problems. However for the sake of completeness we sketch the second method which starts with the representation of the parabolic wave functions [3]

$$
\begin{array}{r}
M_{\kappa, \mu / 2}(a t) M_{\kappa, \mu / 2}(b t)=\frac{t(a b)^{1 / 2}[\Gamma(1+\mu)]^{2}}{\Gamma((1+\mu) / 2+\kappa) \Gamma((1+\mu) / 2-\kappa)} \\
\cdot \int_{0}^{\pi} \exp \left[\frac{-(a+b) t}{2} \cos \phi\right] J_{\mu}\left(t(a b)^{1 / 2} \sin \phi\right)(\cot \phi / 2)^{2} d \phi
\end{array}
$$

and the expansion [4]

$$
(\sin \alpha \sin \beta)^{-\mu} J_{\mu}(z \sin \alpha \sin \beta) \exp (i z \cos \alpha \cos \beta)=2^{2 \mu}(2 \pi z)^{-1 / 2}
$$

$$
\cdot[\Gamma(\mu+1 / 2)]^{2} \sum_{n=0}^{\infty} \frac{i^{n} n !(2 n+2 \mu+1)}{(n+2 \mu+1) z^{\mu}} J_{n+\mu+1 / 2}(z) C_{n}^{\mu+1 / 2}(\cos \alpha) C_{n}^{\mu+1 / 2}(\cos \beta)
$$

where the $C_{n}^{\mu+1 / 2}$ are the Gegenbauer polynomials. If we substitute (8) into (7), integrate termwise and use some of the usual transformations of hypergeometric functions we get (2) with the coefficients given by (6).

3. Inversion of (2). The functions $F_{n+\mu}^{\mu}(w)$ form an orthogonal set with a weight factor. Precisely we have

$$
\begin{aligned}
\frac{\sin \pi \mu}{2 \mu} \int_{-\infty}^{\infty} \frac{F_{n+\mu}^{\mu}(-i t) F_{m+\mu}^{-\mu}(i t) d t}{\cosh \pi t+\cos \pi \mu} & =0, & & n \neq m \\
& =\frac{1}{(2 n+2 \mu+1)}, & & n=m .
\end{aligned}
$$

${ }^{2}$ This was pointed out to one of the authors by A. Erdélyi. 
This result is due to Bateman [5], ${ }^{3}$ and was established for $F_{n}^{\mu}$ with $n$ integral. Extension of the result to $F_{n+\mu}^{\mu}$ can be made without difficulty. By means of (9) we invert (2) and obtain

$$
\begin{aligned}
& \frac{\sin \pi \mu}{2 \mu} \int_{-\infty}^{\infty} \frac{F_{n+\mu}^{-\mu}(-i t) F_{\mu}^{\mu}(i t) \psi(\xi, \lambda) \psi(\eta,-\lambda) d t}{\cosh \pi t+\cos \pi \mu} \\
& \quad=\Gamma(2 \mu+2) \Gamma(n+2 \mu+1)\left(i^{n} / n !\right) k^{-\mu} j_{n+\mu}(k r) P_{n+\mu}^{-\mu}(\cos \theta),
\end{aligned}
$$

a result which is equivalent to one given by Buchholz [3].

4. Miscellaneous results. There are several interesting special cases of (2) and (3) as well as some known results which follow from (2) and the properties of the Pasternack functions. Of these we mention without proof:

(1) For $\mu=m$ an integer, we have from (3)

$$
\psi(\xi, \lambda)=\sum_{n=m}^{\infty} \frac{(2 n+1)(n+m) ! i^{n-m}}{m !(n-m) ! k^{m}} \frac{F_{n}^{m}(w)}{F_{m}^{m}(w)} j_{n}\left(k \xi^{2} / 2\right) .
$$

(2) For $-w=2 s+1+m, s$ and $m$ integers, the expansion (2) reduces to that of Hochstadt [1].

(3) If we put $\mu=m$ in (2) and let $k \rightarrow 0$, we get after some simplifying

$$
J_{m}\left(\lambda^{1 / 2} \xi\right) J_{m}\left(i \lambda^{1 / 2} \eta\right)=\sum_{n=m}^{\infty} \frac{i^{m}(-\lambda r / 2)^{n}}{n !(n+m) !} P_{n}^{m}(\cos \theta)
$$

which may be thought of as an expansion of parabolic potential functions in terms of spherical potential functions.

(4) Finally we sketch a derivation of the bilinear generating function in the continuous case for the parabolic wave functions. The result, due to Erdélyi [6], is

$$
\begin{aligned}
& \frac{(t x y)^{1 / 2}}{1+t} \exp [-(x+y)(1-t) / 2(1+t)] J_{2 \mu}\left[2(t x y)^{1 / 2} /(1+t)\right] \\
& \quad=\frac{1}{2 \pi i} \int_{L} \frac{t^{2} \Gamma(1 / 2-z+\mu) \Gamma(1 / 2+z+\mu)}{[\Gamma(2 \mu+1)]^{2}} M_{z, \mu}(x) M_{z, \mu}(y) d z
\end{aligned}
$$

where $L$ is a path from $-i \infty$ to $i \infty$ separating the poles of $\Gamma(1 / 2-z+\mu)$ from those of $\Gamma(1 / 2+z+\mu)$. To give a derivation of (13) based on the properties of the Pasternack functions, we start with equation (8) and the following representation of the Legendre functions [2]:

\footnotetext{
3 The result given in [5] omits the factor $\sin \pi \mu / 2 \mu$.
} 
(14) $2 \Gamma(\mu+1) e^{\mu x} \operatorname{sech} x P_{n+\mu}^{-\mu}(\tanh x)=\int_{-\infty}^{\infty} e^{i x z} F_{n+\mu}^{\mu}(i z-\mu) \operatorname{sech} \frac{\pi z}{2} d z$.

We now express the Gegenbauer polynomials in (8) in terms of Legendre functions and use the representation (14) for $P_{n+\mu}^{\mu}(\cos \alpha)$ with $\cos \alpha=\tanh x=(1-t) /(1+t)$. Interchanging integration and summation together with the use of (2) yields, after some manipulation, the result (13). The interchange of integration and summation can be justified without difficulty.

\section{REFERENCES}

1. H. Hochstadt, A relationship between parabolic and spherical functions, Proc. Amer. Math. Soc. vol. 8 (1957) pp. 489-491.

2. S. Pasternack, A generalization of the polynomial $F_{n}(x)$, Phil. Mag. vol. 28 (1939) pp. 209-226.

3. H. Buchholz, Die konfluente hypergeometrische Funktion, Berlin, SpringerVerlag, 1953, pp. 85 and 185.

4. G. N. Watson, Theory of Bessel functions, New York, Macmillan, 1934, p. 37.

5. H. Bateman, An orthogonal property of the hypergeometric polynomial, Proc. Nat. Acad. Sci. U.S.A. vol. 28 (1942) pp. 371-375.

6. A. Erdélyi, Generating functions of certain continuous orthogonal systems, Proc. Roy. Soc. Edinburgh vol. 61 (1941) pp. 61-70.

Michigan State University 